Research Article

\title{
HPLC/MS-MS Identification of Oak Quercus aegilops Root Tannins
}

\author{
Ayed S. Amr $\mathbb{D}^{1},{ }^{1}$ Mousa N. Ahmad $\mathbb{D}^{1},{ }^{1}$ Jalal A. Zahra $\mathbb{D D}^{2}$ and Mai A. Abdullah $\mathbb{D}^{3}$ \\ ${ }^{1}$ Department of Nutrition and Food Technology, University of Jordan, Amman 11942, Jordan \\ ${ }^{2}$ Department of Chemistry, University of Jordan, Amman 11942, Jordan \\ ${ }^{3}$ Al-Balqa Applied University, Huoson, Irbid, Jordan \\ Correspondence should be addressed to Ayed S. Amr; ayedamr@ju.edu.jo
}

Received 2 September 2020; Revised 31 January 2021; Accepted 18 February 2021; Published 2 March 2021

Academic Editor: Suresh Kannan Balasingam

Copyright (C) 2021 Ayed S. Amr et al. This is an open access article distributed under the Creative Commons Attribution License, which permits unrestricted use, distribution, and reproduction in any medium, provided the original work is properly cited.

Tannins are natural polyphenolic compounds widely distributed in the plant kingdom in the leaves, bark, fruits, and other parts. They have various biological functions in humans and animals and are used mainly in the pharmaceutical and cosmetic industries. The aim of this work was to isolate, extract, purify, and identify the tannins from the root bark of a common oak tree (Quercus aegilops L.) in Jordan and around the Mediterranean. The results showed that at least one form of ellagitannin (ellagic acid ester), quercitrin, afzelechin, valoneic acid, trigalloyl glucose, and catechin was identified in addition to two unidentified compounds. Results of this work can help in developing an ESI MS/MS search library for the constituents of the tannins of oak (Quercus aegilops L.) root bark.

\section{Introduction}

Tannins are complex heterogeneous group of polyphenolic secondary metabolites of higher plants which share the ability to bind and precipitate proteins, alkaloids, and polysaccharides [1]. Their molecular weights range between 500 and 20,000 Da. [2], although Okuda and Ito [3] reported that some types of tannins have molecular weights smaller than $500 \mathrm{Da}$. They are considered to be free radical scavengers [4] which also have the ability to inactivate microbial adhesions as well as enzymes, and cell-envelop and transport proteins [5]. Widsten et al. have shown that tannins have antimicrobial properties due to their bacterial toxicity exerted by their $o$-diphenol groups which act as iron chelators [6]. Hydroxyl groups in tannins may also play an important role in the molecule's ability to permeate the cell walls of bacteria [6]. It also was reported that the hydrolyzed tannins "ellagitannins" have antimicrobial activity against fungi, viruses, and bacteria including those antibiotic-resistant strains [7].

Many studies have shown that polyphenols have anticarcinogenic effect as well $[8,9]$. Romani et al. [10] have reported that condensed tannins can interact with biological systems by playing antioxidant, antiallergy, antihypertensive, and antimicrobial roles [10]. The same authors have pointed out that tannic acid possesses antimutagenic, anticancer, and antioxidant properties. There is also evidence that phenolic compounds exert an obesity-preventing effect through inhibition of the pancreatic lipase [11]. Dietary polyphenols may repress growth of the adipose tissue through their antiangiogenic activity and by modulating adipocyte metabolism; accordingly, they may have benefits in controlling obesity $[9,11]$. Matsumoto and Yokoyama [12] have reported that tannins have the ability to reduce plasma cholesterol levels accompanied by acceleration of fecal lipid excretion and bile acid excretion in cholesterol-fed rats. Recent studies have shown that the antioxidant activity of hydrolyzed tannins may reduce serum cholesterol and triglycerides and suppress lipogenesis induced by insulin [10]. It is well established that the composition and concentration of tannins vary depending on species, part, and age of the plant from which they are extracted [1].

Based on their specific structures and chemical properties, tannins are classified into hydrolysable, condensed, and complex $[13,14]$. Hydrolysable tannins, which are found in many plant species including oak, sumac, and chestnuts 
are subclassified into gallotannins and ellagitannins [15]. Conversely condensed tannins (proanthocyanidins) are oligomeric or polymeric flavonoids consisting of flavan-3-ol (catechin or epicatechin) units linked either via C4-C6 or C4-C8 bonds (B type proanthocyanidins) [16, 17]. Gallic acid esters may also be found in tannins [8]. Condensed tannins were extracted from quebracho wood and mimosa bark [8], as well as grape seeds [17]. Complex tannins consist of flavan-3-ol units (catechin moiety) glycosidically bound to gallotannin or ellagitannin units. Additionally, they have been detected in various oak species (e.g., Quercus petraea) $[1,18,19]$.

A number of methods have been proposed for quantitative analysis of tannins; these methods are classified into general phenolic methods, functional group methods, and chromatographic (HPLC and UPLC) and protein precipitation methods [14, 20-25]. Liu and White recommended the use of HPLC, nuclear magnetic resonance (NMR), mass spectroscopy (MS), infrared (IR), and gas chromatography (GC) for the identification of tannins [17]. Williamson and Carughi [26] recommended the use of HPLC with mass spectrometric detection and appropriate isotopically labeled standards for the analysis of phenols in general and tannins in particular.

The aim of this work is to extract tannins from oak (Quercus aegilops) root bark, purify them on Sephadex LH20 column, and identify them using HPLC/MS-MS technique as this system is supplied with a library which facilitates the identification of the different tannin compounds.

\section{Materials and Methods}

2.1. Collection and Preparation of Oak Root Bark. Oak root samples of Quercus aegilops L. were collected in November 2013 from an oak forest near Amman/Jordan, washed with distilled water, and air-dried at room temperature for two weeks. The bark was stripped off and separated from the heartwood ground using hummer mill and stored in air tight, low-density polyethylene bags in a refrigerator for further analysis.

2.2. Extraction of Tannins. Ground oak root bark powder was extracted as described by Hagerman [21] with $80 \%$ (v/ v) ethanol: water. One gram of the oak root powder was suspended in $10 \mathrm{ml}$ of $80 \%(\mathrm{v} / \mathrm{v})$ ethanol, stirred well, and allowed to settle overnight in a refrigerator. The extract was then removed and filtered through Whatman \#40 filter paper. The filtrate was mixed with $100 \mathrm{ml}$ of Sephadex LH20 slurry (25 gm of Sephadex LH20 (SigmaAldrich LH20100) prepared as a slurry in $100 \mathrm{ml}$ of $80 \%$ $(\mathrm{v} / \mathrm{v})$ ethanol and kept overnight in the refrigerator [21] to be equilibrated later with $95 \%(\mathrm{v} / \mathrm{v})$ ethanol, stirred for 3 minutes, and filtered through a coarse glass sintered funnel; a brown color was developed on Sephadex LH20 beads. Sephadex LH20 is commonly used to separate tannins from nontannin small molecular weight compounds, as it absorbs tannins in alcohol and releases them in aqueous acetone solution [21]. The Sephadex LH20 beads were then washed with $95 \%$ ethanol until the absorbance of the washings at $280 \mathrm{~nm}$ was around zero with colorless elution to equilibrate the Sephadex. Finally, the Sephadex was washed to elute tannins with $50 \%(\mathrm{v} / \mathrm{v})$ aqueous acetone until the beads became clear white. All acetone washings were combined, then the acetone was removed under vacuum at a temperature $\leq 30^{\circ} \mathrm{C}$, and the remaining aqueous solution was extracted three times with equal volumes of ethyl acetate. The lower aqueous phase containing the tannins was kept, traces of ethyl acetate were removed by evaporation at room temperature, and the aqueous sample was freeze-dried (Operon FDB 5502) into a fluffy brown powder, weighed and kept frozen.

\subsection{HPLC/MS-MS Chromatography of Oak Tannins}

2.3.1. HPLC Instrumentation. Chromatographic separations for different phenolic compounds extracted with ethanol and purified on Sephadex LH20 were performed on a reversed phase HPLC. An Agilent Eclipse XDB HPLC system was used. C18 column $(150 \times 4.6 \mathrm{~mm}, 5 \mu \mathrm{m})$ using an Agilent 1200 LC system (Agilent, Santa Clara, CA, USA) was equipped with degasser (G1379 B) and binary pump (G1312 A) along with autosampler (G1367 B). The autosampler was maintained at $6^{\circ} \mathrm{C}$ and programmed to draw $5 \mu \mathrm{l}$ of sample for chromatographic separation. The method was validated using an isocratic mobile phase of deionized water $/ 0.01 \%$ trifluoroacetic acid (TFA) in methanol $(30: 70, \mathrm{v} / \mathrm{v})$ applied at a flow rate of $0.70 \mathrm{ml} /$ min. The column temperature was kept at $27^{\circ} \mathrm{C}$. The total analytical run time was $10.0 \mathrm{~min}$ for each sample. Detection was carried out on an AB Sciex (Applied Biosystem/MDS SCIEX, Foster City, CA, USA) API-3200 Q-Trap mass spectrometer, equipped with a Turboionspray interface operated in negative ion mode-ESI (electrospray ionization). Separated compounds were scanned using the multiple reaction monitoring (MRM) method. Optimized instrument parameters were medium-flow collision-activated dissociation (CAD) gas with curtain (CUR) gas: 24 psi; nebulizer gas (gas1): 30 psi; heater gas (gas 2): 25 psi; ion spray voltage: $-4500 \mathrm{~V}$; source temperature: $550^{\circ} \mathrm{C}$. Compound-dependent voltage parameters are as listed in Table 1. System control and data analysis were performed by $\mathrm{AB}$ Sciex Analyst software (version 1.5). The following set of standards was used: catechin (C1251 Sigma), gallic acid (G7384 Sigma), ellagic acid (E2250 Sigma), taxifolin (78666 Sigma-Aldrich), quercetin (Q4951 Sigma-Aldrich), p-coumaric acid, and ferulic acid (ICN-Biomed). The different tannin compounds were confirmed by their MS-MS profiles after ion isolation using linear ion trap (LIT) in comparison with the corresponding pure standards. The enhanced MS screening method was used to screen and confirm tannin products of gallotannins and ellagitannins as shown in Table 2. Also, in order to measure the expected large compounds, screening was optimized from 90-1500 Atomic Mass Units (amu). 
TABLE 1: Compound-dependent voltage parameters.

\begin{tabular}{lccccc}
\hline \multirow{2}{*}{ Analyte } & \multicolumn{4}{c}{ MS-MS parameters } \\
& $\mathrm{Q}^{1}$ & $\mathrm{Q}^{2}$ & $\mathrm{DP}^{3}$ & $\mathrm{EP}^{4}$ & $\mathrm{CE}^{5}$ \\
\hline Ellagic acid & 300.6 & 200.9 & -64 & -5 & -42 \\
Gallic acid & 168.8 & 124.9 & -50 & -5 & -20 \\
Quercetin & 300.9 & 150.9 & -50 & -5 & -27 \\
p-Coumaric acid & 162.7 & 118.8 & -33 & -5 & -20 \\
Ferulic acid & 192.9 & 133.9 & -35 & -6 & -1 \\
Propyl gallate & 210.6 & 124 & -60 & -5 & -2 \\
Pyrocatechol & 108.7 & 90.9 & -63 & -5 & -35 \\
Catechin & 288.8 & 230.8 & -40 & -3 & -29 \\
Taxifolin & 302.8 & 230.8 & -32 & -3 & -24 \\
\hline
\end{tabular}

${ }^{1} \mathrm{Q} 1$ : parent. ${ }^{2} \mathrm{Q} 3$ : daughter. ${ }^{3} \mathrm{DP}$ : declustering potential. ${ }^{4} \mathrm{EP}$ : entrance potential. ${ }^{5} \mathrm{CE}$ : energy of collision. ${ }^{6} \mathrm{CXP}$ : cell exist potential.

TABLE 2: Selected group of gallotannins and ellagitannins screened by enhanced MS using the LIT.

\begin{tabular}{lcc}
\hline Parent Q1 $(\mathrm{m} / z)$ & Compound & Daughter Q3 \\
\hline 933.4 & Trigalloyl-gallagoyl-glucose & $783.2,631,481.1,301.1$ \\
933.2 & Trigalloyl-HHDP-glucose & $631,569.1,467.2,301.2$ \\
933.1 & Pentagalloyl-glucose & $631.1,569.2,425.1,301.2$ \\
783.4 & Tetragalloyl-glucose & $764.1,746,481,301.1$ \\
783.2 & Tetragalloyl-glucose & $763.1,481,301$ \\
933.4 & Triagalloyl-glucose & $613.1,481,301.1$ \\
783.6 & Tetragalloyl-glucose & $764.4,651.1,481,301.1$ \\
933.1 & Triagalloyl-glucose & $613.1,481,301.1$ \\
783 & Tetragalloyl-glucose & $764.0,746,481,301.1$ \\
613.5 & Dehydrated tergallic-C-glucoside & $595.5,523.6,493.2,301.1$ \\
613.7 & Dehydrated tergallic-C-glucoside & 493.1 \\
933.2 & Trigalloyl-glucose & $614.1,467.7,301.1$ \\
631.4 & Tergallic-O-glucoside & $628.1,451.1,301.1$ \\
469.4 & Valoneic acid dilactone & $425.1,300.9$ \\
469.0 & Valoneic acid dilactone & $425.9,300.9$ \\
469.2 & Valoneic acid dilactone & $424.9,300.9$ \\
595.5 & Identify unknown & $463.3,301$ \\
927.4 & Valoneic acid dimer & $463.3,301$ \\
933.3 & Trigalloyl-HHDP-glucose & $756.6,463.2,301.1$ \\
867.6 & Ellagic acid pentoside dimer & 433,301 \\
850.0 & Identify unknown & $821.1,804.4,677.7,451.5,301.1$ \\
\hline
\end{tabular}

2.4. Tannin Compound Identification. The different compounds in the tannin extract were identified by their MS-MS profiles after ion isolation using linear ion trap, in comparison with the corresponding pure standards. The enhanced MS screening method was used to screen for tannin products of gallotannins as shown in Table 2. Also, in order to measure the expected large compounds, screening was optimized from 90-1500 amu on negative ion mode using the API-3200 Q-Trap mass spectrometer.

\section{Results and Discussion}

3.1. HPLC/MS-MS of Oak Tannin Extracts. To qualify tannin compounds extracted from the oak Quercus aegilops root bark, a multiple reaction monitoring (MRM) method using HPLC/ ESI-MS negative mode was developed and optimized. The chromatographic conditions were optimized using a multiple set of available standards (Figure 1), and the extracted ion chromatograms (XICs) of MRM for the tannin extract were developed. The results are summarized in Table 3, where the gallic acid, catechin, and ellagic acid were detected.

Sephadex extracts were characterized with the presence of ellagic acid, gallic acid, and catechin (Figure 2). Retention time for each of the extracted compounds was determined; however, retention time of extracted compounds was so close to each other, reflecting the main limitation in characterizing of tannins due to the large number of molecules under the same chemical class. On the other hand, they might be present in isomers which implicate the chromatographic separation process, and also, isomerization or depolymerization can take place due to environmental conditions [8]. Furthermore, tannin derivatives were not completely separable by chromatography and cannot be distinguished through their MS due to their similar structures such as the catechin and epicatechin [8]. Enhanced MS screening method was also used to screen some of the expected tannin products. Table 4 presents our observations. MRM extract results are shown in Figure 3. 


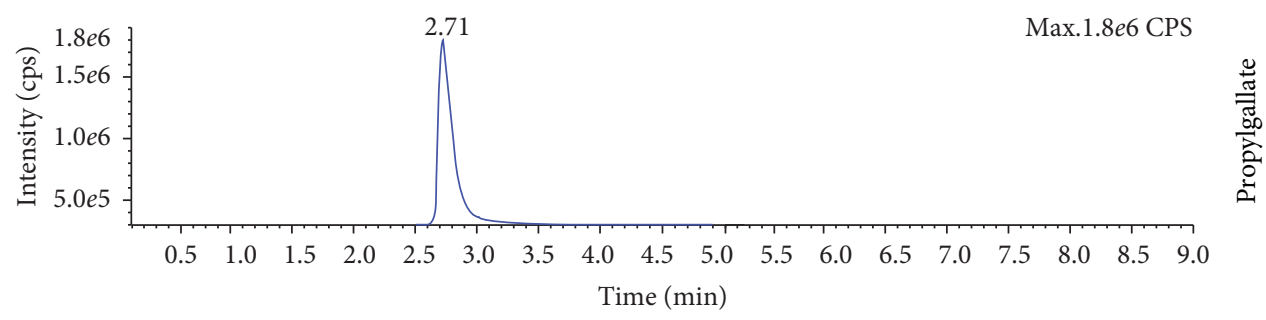

XIC of- MRM (13 paris): 210.6124.0/ Da ID:propylgallate from Sapmle 1 (standard mix) of 27.02.2014.wiff (Turbo spray)

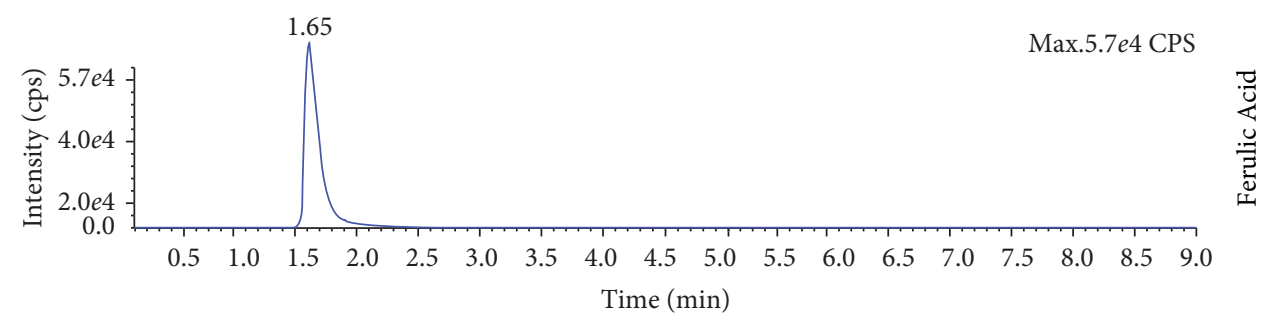

XIC of- MRM (13 paris): 192.9 / 133.9 Da ID:ferulic Acid from Sapmle 1

(standard mix) of 27.02.2014.wiff (Turbo spray)

Max.1.3e5 CPS

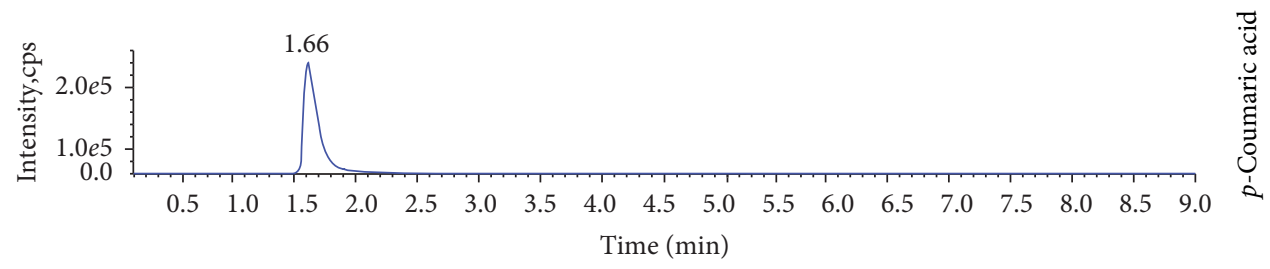

XIC of- MRM (13 paris): 162.7/ 118.8 Da ID:p-Coumaric acid from Sapmle 1 (standard mix) of 27.02.2014.wiff (Turbo spray)

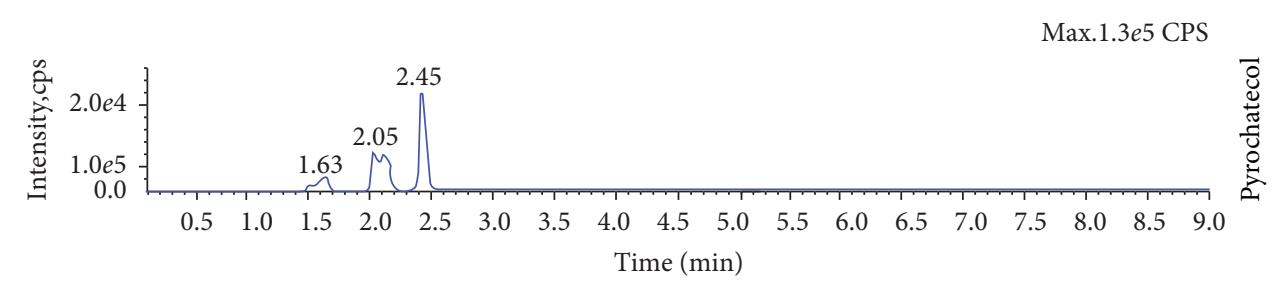

XIC of- MRM (13 paris): 108.7 / 90.9 Da ID:Propylgallate from Sapmle 1 (standard mix) of 27.02.2014.wiff (Turbo spray)

(a)

FIGURE 1: Continued. 


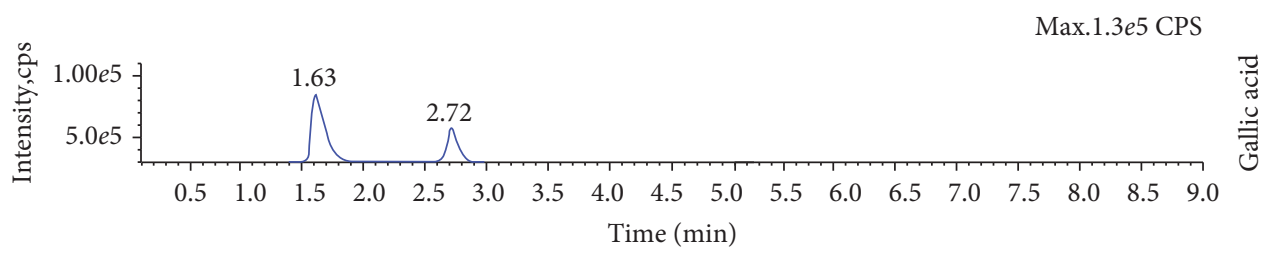

XIC of- MRM (13 paris): 168.8/ 124.9 Da ID:gallic acid from Sapmle 1

(standard mix) of 27.02.2014.wiff (Turbo spray), Smoothed

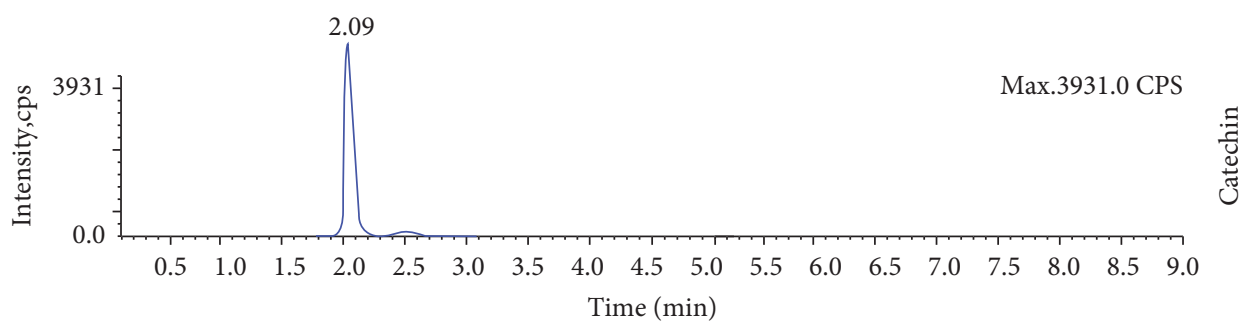

XIC of- MRM (13 paris): 288.8/ 183.0 Da ID:catechin from Sapmle 1

(standard mix) of 27.02.2014.wiff (Turbo spray), Smoothed

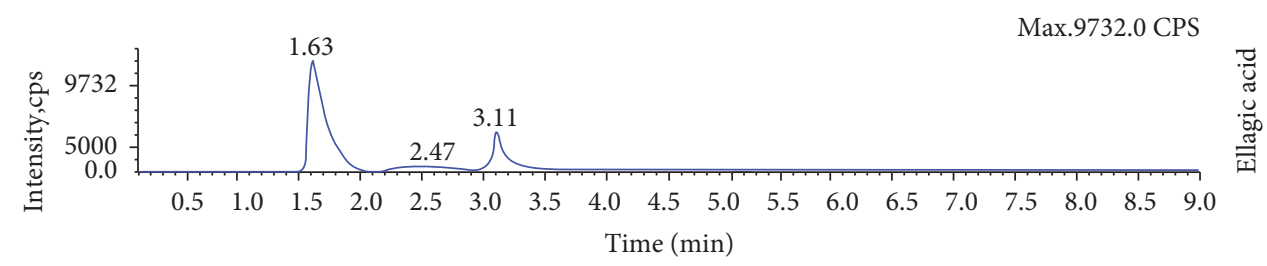

XIC of- MRM (13 paris): 300.6/ 200.9 Da ID:ellagic acid from Sapmle 1

(standard mix) of 27.02.2014.wiff (Turbo spray), Smoothed

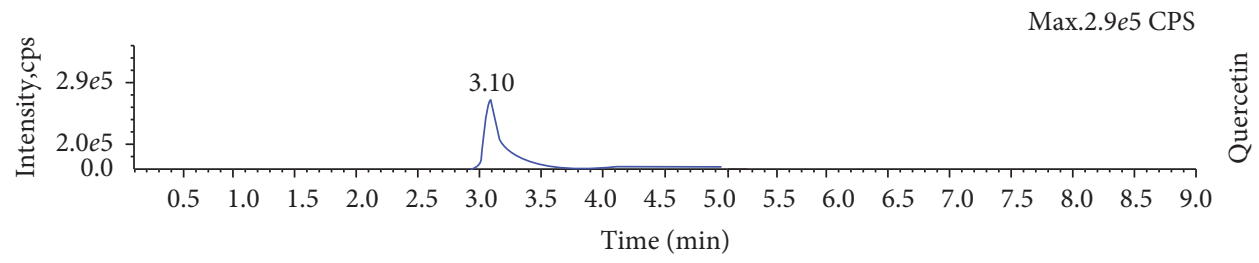

XIC of- MRM (13 paris): 300.9/ 150.9 Da ID:quercetin from Sapmle 1 (standard mix) of 27.02.2014.wiff (Turbo spray)

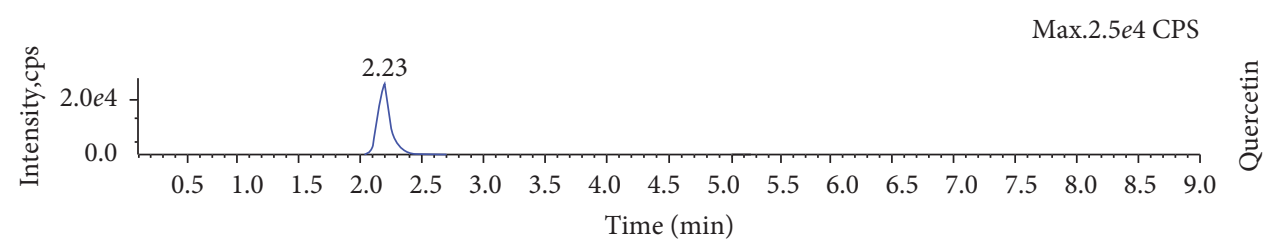

XIC of- MRM (13 paris): 302.8/ 230.8 Da ID:taxifolin from Sapmle 1 (standard mix) of 27.02.2014.wiff (Turbo spray), Smoothed

(b)

FIGURE 1: Standard mix of HPLC/MS-MS (optimized instrument parameters: medium-flow collision-activated dissociation (CAD) gas wiht curtain (CUR) gas: 24 psi; nebulizer gas (gas 1): 30 psi; heater gas (gas 2): 25 psi; ion spray voltage: $-4500 \mathrm{~V}$; source temperature: $550^{\circ} \mathrm{C}$; compound-dependent voltage parameters for each standard are shown in Table 3.

TABLE 3: Recorded retention times of identified compounds, where standards were available.

\begin{tabular}{lc}
\hline Compound & $\mathrm{RT}_{\min }$ \\
\hline Gallic acid & 1.59 \\
Catechin & 1.70 \\
Ellagic acid & 1.57 \\
\hline
\end{tabular}

$\mathrm{RT}_{\min }$ : retention time in min.
In order to measure the expected large compounds, a full MS screening was optimized from 90 to $1500 \mathrm{amu}$ on negative ion mode using the API-3200 Q-Trap mass spectrometer (Figure 4) extracted MS-MS scan. There was no current available library for polyphenols/tannin searching for the ESI, accordingly the spectral identifications were made by comparing the parent ion (Q1) molecular weight 
Max.3562.4 CPS

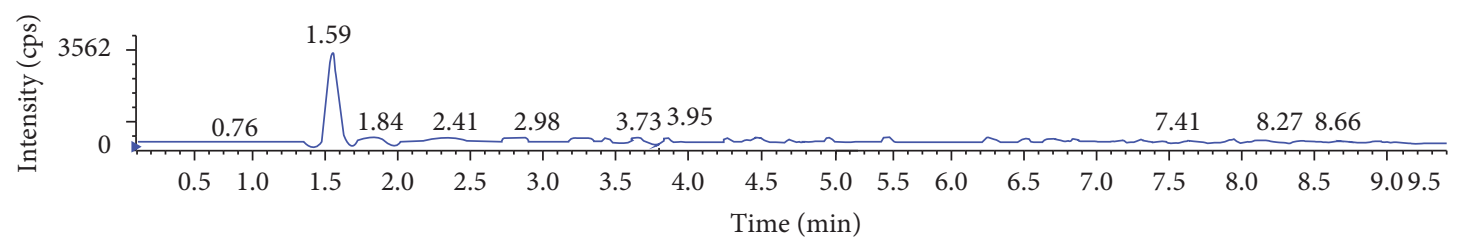

XIC of- MRM (13 paris): 168.8/ 124.9 Da ID:gallic acid from Sapmle 2

(standard mix) of 27.02.2014.wiff (Turbo Spray),Smoothed

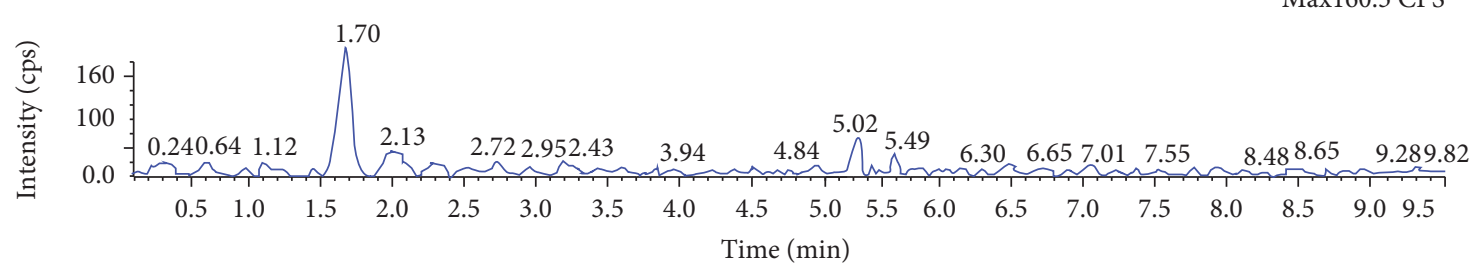

XIC of- MRM (13 paris): 288.8/ 183.0 Da ID:catechin from Sapmle 1

(standard mix) of 27.02.2014.wiff (Turbo spray), Smoothed

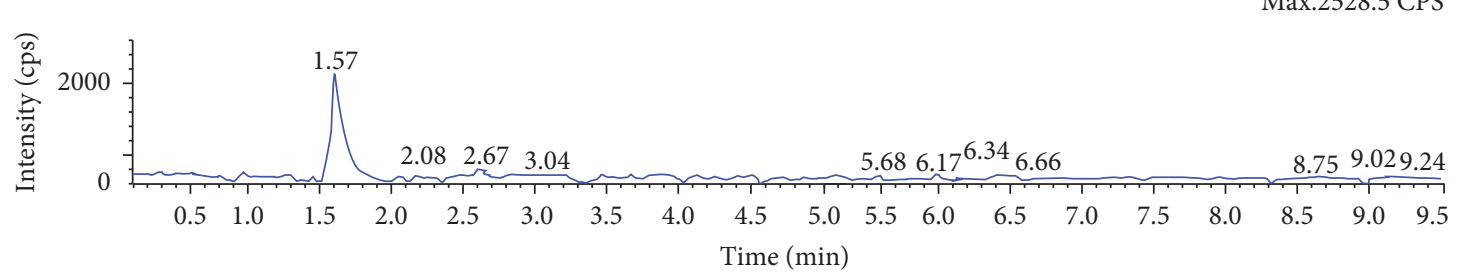

XIC of- MRM (13 paris): 300.6/ 200.9 Da ID:ellagic acid from Sapmle 1

(standard mix) of 27.02.2014.wiff (Turbo spray), Smoothed

Figure 2: The XIC MRM chromatogram of the extracted tannins (from top to bottom, gallic acid, catechin, and ellagic acid).

TABLE 4: $(\mathrm{m} / z)$ compound voltage dependent parameters detected.

\begin{tabular}{lccc}
\hline $\mathrm{Rt}_{\min }$ & Parent Q1 $(\mathrm{m} / z)$ & Compound & Daughter Q3 \\
\hline 6.18 & 867.6 & Ellagic acid pentoside dimer & $434.5,301$ \\
11.52 & 447.4 & Quercitrin & $315.4,153.4$ \\
15.94 & 545.3 & Afzelechin & $528,483.7$ \\
17.46 & 473.3 & Valoneic acid & $448.7,316.5,301.6$ \\
19.2 & 934 & Trigalloyl-glucose & 626.5 \\
15.62 & 595.5 & Unknown & No fractions \\
15.55 & 1083 & Unknown & No fractions \\
\hline
\end{tabular}

with those obtained from literature reports, or searching the online MS-libraries. From the extracted chromatograms (Figure 4), a group of compounds were identified, as indicated in Table 2.

It was observed that Sephadex LH20 extracted tannins contained at least one form of an ellagitannin/ellagic acid esters/galloyl-glucose, ellagic acid, in addition to gallic acid, valoneic acid, catechin, and afzelechin (condensed tannin). Also, there were a number of compounds with molecular weights of 595.5 and 1083 that were characterized as unknown tannins. Unfortunately, although the retention time was identified, the MS fraction was not detected.

Mammela et al. [19] investigated tannins from two types of oak (the European and American species) and reported that both species had glucose gallic and ellagic acid esters, grandinin/roburin $E$, castalagin/vescalagin, gallic acid, valoneic acid bilactone, monogalloyl glucose, digalloyl glucose, trigalloyl glucose, ellagic acid rhamnose, quercitrin, and ellagic acid.

A study of Cantos et al. [27] on acorns of some Quescus species (Q. rotundifolia, Q. ilex, and Q. suber) has shown that thirty-two different phenolic compounds were quantified. They were gallic acid derivatives, in the form of either galloyl esters of glucose or ellagic acid derivatives in addition to valoneic acid.

Using both methods of HPLC/mass spectroscopy and MNR, Glabasnia and Hofmann [28] reported a number of ellagitannins and their derivatives in Quercus alba L. and Quercus robur L. including grandinin, roburin $E$, vescalagin castalagin, 33-deoxy-33 carboxyvescalagin, 

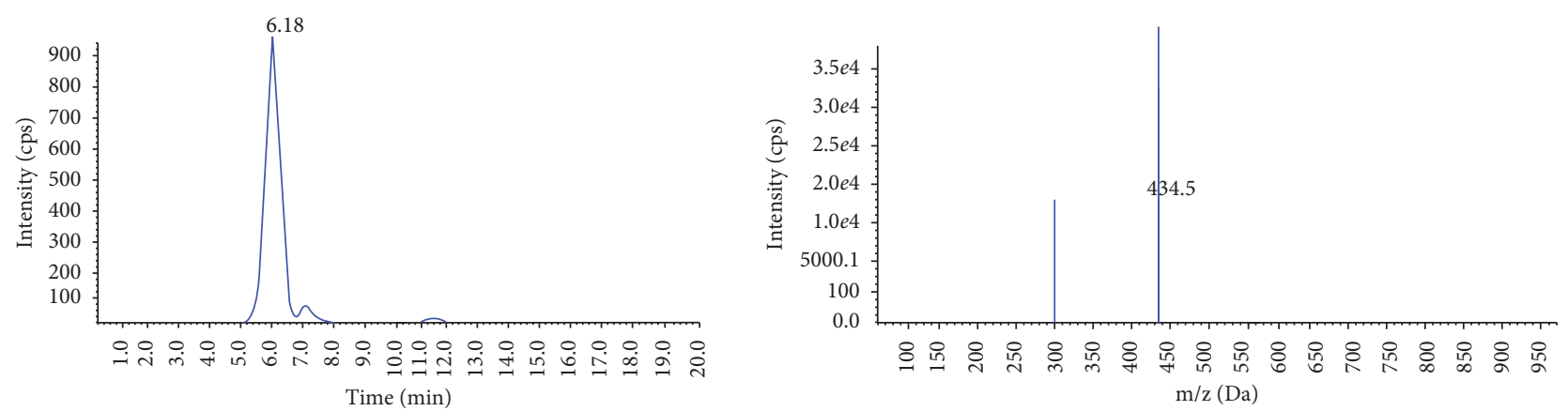

Ellagic acid pentoside dimer
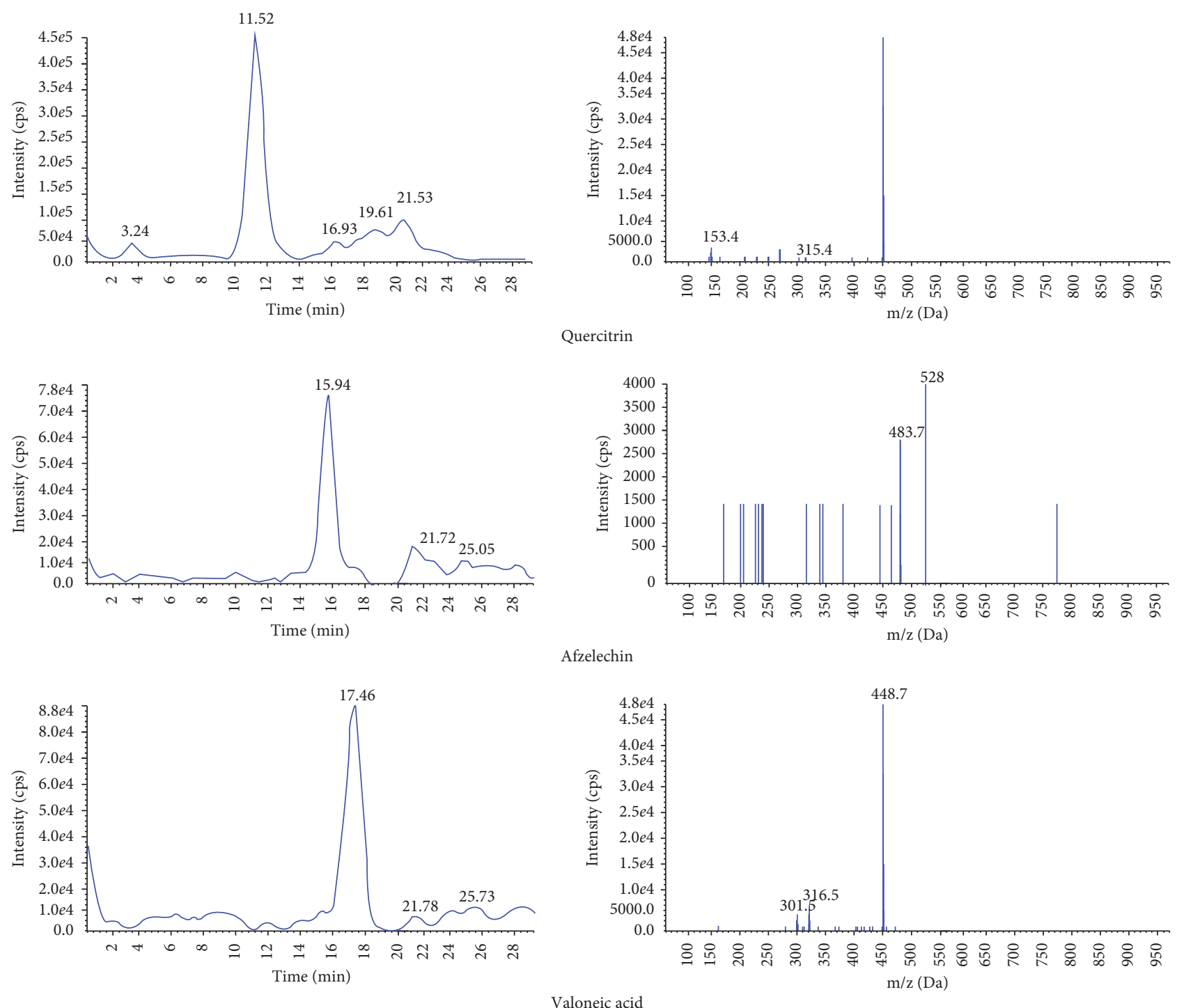

(a)

Figure 3: Continued. 


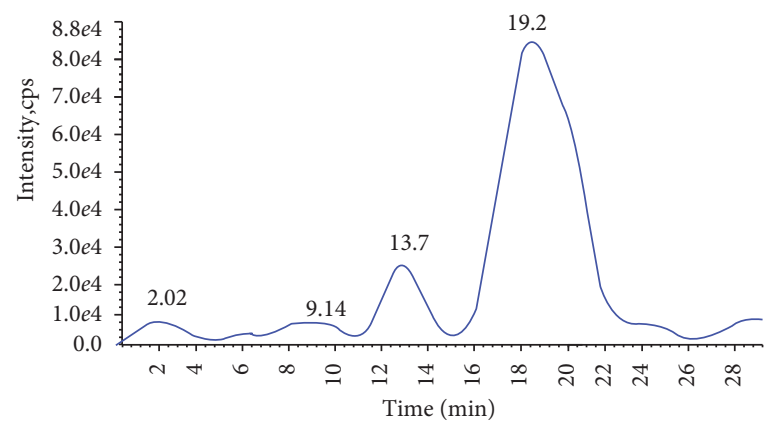

Trigalloyl-glucose

(b)

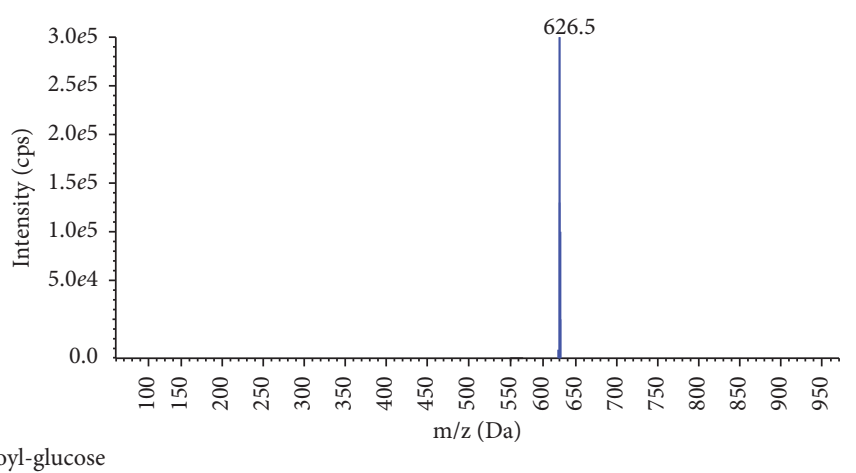

FIGURE 3: MRM MS-MS results.

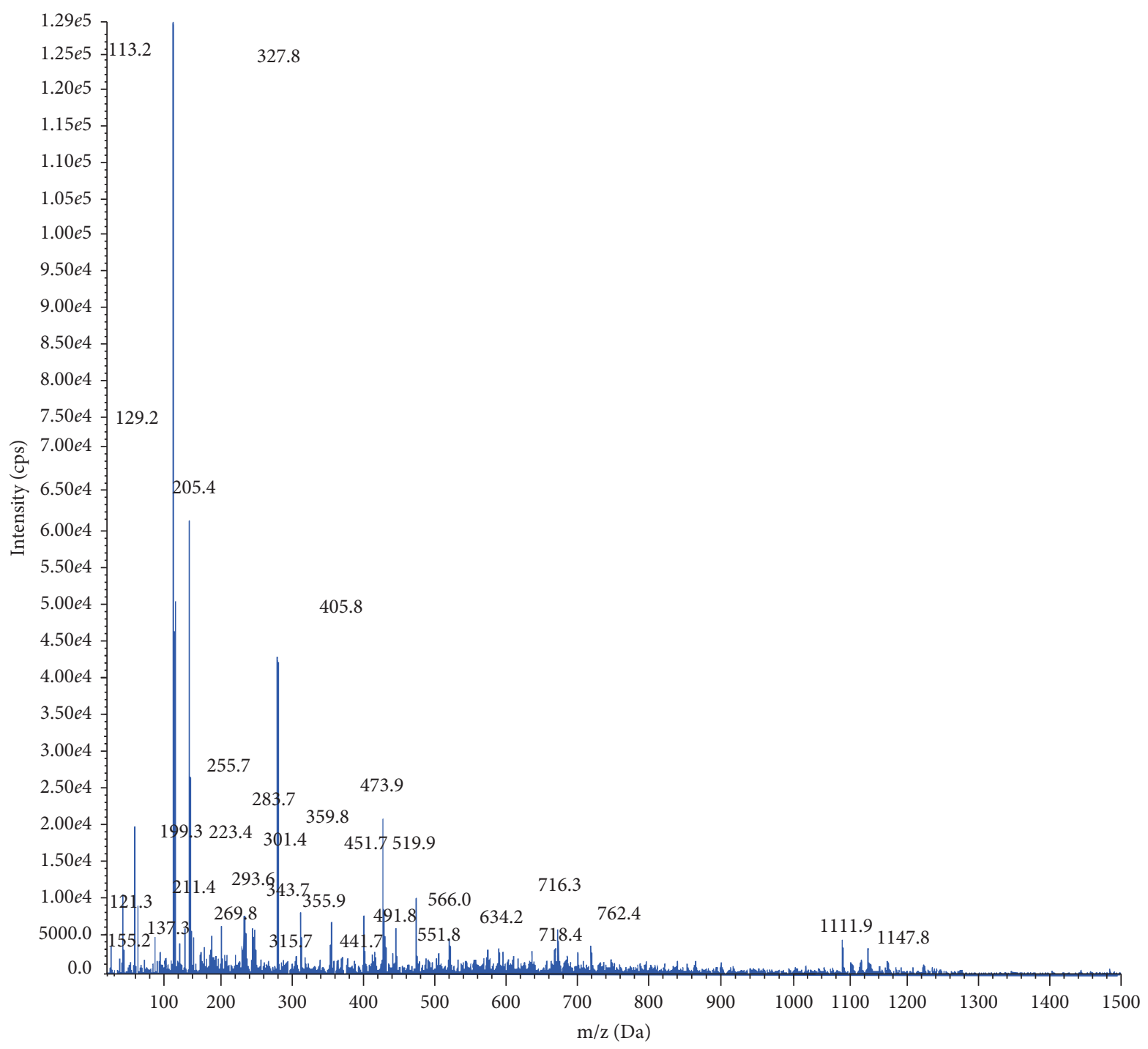

-EMs :Exp1, 0.031 to $20.260 \mathrm{~min}$ from Sample 10(ethanol extract) of27.02.2014.wiff (Turbo Spray), Subtracted < - EMS:Exp1,0.0...

Figure 4: MS-MS scan results. 
roburin $\mathrm{A}$, roburin $\mathrm{B}$, roburin $\mathrm{C}$, pentagalloyl-â-d-glucose, ellagic acid, gallic acid, epigallocatechin 3-gallate, and caffeine.

Our results were in line with some of the findings by Mammela et al. [19], Cantos et al. [27], and Glabasnia and Hofmann, [28]. Application of the HPLC/ESI-MS in the identification tannins of Quercus aegilops L. species revealed the presence of glucose gallic and ellagic acid esters, gallic acid, valoneic acid, trigalloyl glucose, quercitrin, and ellagic acid.

Masson et al. [29] reported monomers of ellagitannins; vescalagin and castalagin represented a substantial quantity of the total amount of ellagitannins present in oak. In this study, both monomers and dimmers of ellagitannins were screened qualitatively albeit quantitative analysis was not attained. Vescalagin and castalagin may undergo hydrolysis or participate in nonspecific reactions in presence of oxygen leading to changes in ionic strength and higher temperature $\left(60^{\circ} \mathrm{C}\right)[30,31]$. Season variations also affect the content and stability of vescalagin, castalagin, and roburins because of the reactions of polymerization or hydrolysis [31]. This perhaps the reason that our results did not show vescalagin and castalagin in analyzed tannins.

Contrary to the findings of Mammela et al., Cantos et al., and Glabasnia and Hofmann, $[19,27,28]$ grandinin/roburin, castalagin/vescalagin, and valoneic acid bilactone were not detected. This may be attributed to differences in the technical method of analysis, differences in the oak species, part of plant from which the tannin extracted, seasonal changes, and method of extraction, or it may attribute to the sensitivity of our instrumental techniques. Moreover, a possible wide variety of isomeric structures in the tannins makes an absolute separation and confirmation difficult.

To the best of our knowledge, there were no previous studies on screening and characterization of tannin compounds extracted from the roots Quercus aegilops, and our results bring a new insight into tannin characterization of this tree species.

\section{Data Availability}

The data were generated and analyzed in the department of Nutrition and Food Technology/College of Agriculture/ University of Jordan.

\section{Conflicts of Interest}

The authors declare that they have no conflicts of interest.

\section{Acknowledgments}

This work was supported by a grant from the deanship of scientific research at the University of Jordan/Amman/ Jordan.

\section{References}

[1] L. Falcão and M. E. M. Araújo, "Tannins characterisation in new and historic vegetable tanned leathers fibres by spot tests," Journal of Cultural Heritage, vol. 12, no. 2, pp. 149-156, 2011.
[2] W. V. Zucker, "Tannins: does structure determine function? An ecological perspective," The American Naturalist, vol. 121, no. 3, pp. 335-365, 1983.

[3] T. Okuda and H. Ito, "Tannins of constant structure in medicinal and food plants-hydrolyzable tannins and polyphenols related to tannins," Molecules, vol. 16, no. 3, pp. 2191-2217, 2011.

[4] M. A. Shad, H. Nawaz, T. Rehman, H. B. Ahmad, and M. Hussain, "Optimization of extraction efficiency of tannins from Cichorium intybus L.: application of response surface methodology," Journal of Medicinal Plants Research, vol. 6, no. 28, pp. 4467-4474, 2012.

[5] W. Bors and C. Michel, "Antioxidant capacity of flavanols and gallate esters: pulse radiolysis studies," Free Radical Biology and Medicine, vol. 27, no. 11-12, pp. 1413-1426, 1999.

[6] P. Widsten, C. Heathcote, A. Kandelbauer et al., "Enzymatic surface functionalisation of lignocellulosic materials with tannins for enhancing antibacterial properties," Process Biochemistry, vol. 45, no. 7, pp. 1072-1081, 2010.

[7] L. Lipinska, E. Klewicka, and M. Sojka, "Structure, occurrence and biological activity of ellagitannins: a general review," Acta Scientiarum Polonorum Technologia Alimentaria, vol. 13, 2014.

[8] H. Sakagami, Y. Jiang, K. Kusama et al., "Cytotoxic activity of hydrolyzable tannins against human oral tumor cell lines-a possible mechanism," Phytomedicine, vol. 7, no. 1, pp. 39-47, 2000.

[9] M. González-Castejón and A. Rodriguez-Casado, "Dietary phytochemicals and their potential effects on obesity: a review," Pharmacological Research, vol. 64, no. 5, pp. 438-455, 2011.

[10] A. Romani, M. Campo, and P. Pinelli, "HPLC/DAD/ESI-MS analyses and anti-radical activity of hydrolyzable tannins from different vegetal species," Food Chemistry, vol. 130, no. 1, pp. 214-221, 2012.

[11] T. Sergent, J. Vanderstraeten, J. Winand, P. Beguin, and Y.-J. Schneider, "Phenolic compounds and plant extracts as potential natural anti-obesity substances," Food Chemistry, vol. 135, no. 1, pp. 68-73, 2012.

[12] K. Matsumoto and S.-I. Yokoyama, "Induction of uncoupling protein- 1 and -3 in brown adipose tissue by kaki-tannin in type 2 diabetic NSY/Hos mice," Food and Chemical Toxicology, vol. 50, no. 2, pp. 184-190, 2012.

[13] A. Gangwal, "Extraction, estimation and thin layer chromatography of tannins: a review," International Journal of Pharmaceutical and Chemical Sciences, vol. 2, no. 3, pp. 1585-1588, 2013.

[14] I. Fecka, A. Z. Kucharska, and A. Kowalczyk, "Quantification of tannins and related polyphenols in commercial products of tormentil (Potentilla tormentilla)," Phytochemical Analysis, vol. 26, no. 5, pp. 353-366, 2015.

[15] S. G. Sáyago-Ayerdi, C. L. Moreno-Hernández, E. MontalvoGonzález et al., "Mexican "Ataulfo" mango (Mangifera indica L) as a source of hydrolyzable tannins. Analysis by MALDITOF/TOF MS," Food Research International, vol. 51, no. 1, pp. 188-194, 2013.

[16] B. Lorrain, I. Ky, L. Pechamat, and P.-L. Teissedre, "Evolution of analysis of polyhenols from grapes, wines, and extracts," Molecules, vol. 18, no. 1, pp. 1076-1100, 2013.

[17] S. X. Liu and E. White, "Extraction and characterization of proanthocyanidins from grape seeds," The Open Food Science Journal, vol. 6, pp. 5-11, 2012. 
[18] H. P. S. Makkar, "Protein precipitation methods for quantitation of tannins: a review," Journal of Agricultural and Food Chemistry, vol. 37, no. 4, pp. 1197-1202, 1989.

[19] P. Mämmelä, H. Savolainen, L. Lindroos, J. Kangas, and T. Vartiainen, "Analysis of oak tannins by liquid chromatography-electrospray ionisation mass spectrometry," Journal of Chromatography A, vol. 891, no. 1, pp. 75-83, 2000.

[20] N. Silanikove, A. Perevolotsky, and F. D. Provenza, "Use of tannin-binding chemicals to assay for tannins and their negative postingestive effects in ruminants," Animal Feed Science and Technology, vol. 91, no. 1-2, pp. 69-81, 2001.

[21] A. E. Hagerman, "Hydrolyzable tannin structural chemistry," The Tannin Handbook, pp. 1-8, Miami University, Oxford, OH, USA, 2002.

[22] C. Lu, H. Wang, W. Lv et al., "Ionic liquid-based ultrasonic/ microwave-assisted extraction combined with UPLC-MS-MS for the determination of tannins inGalla chinensis," Natural Product Research, vol. 26, no. 19, pp. 1842-1847, 2012.

[23] L. Pinasseau, A. Verbaere, M. Roques et al., "A fast and robust UHPLC-MRM-MS method to characterize and quantify grape skin tannins after chemical depolymerization," Molecules, vol. 21, no. 10, pp. 1409-1422, 2016.

[24] Y. Tong, Y. Jiang, X. Chen et al., "Extraction, enrichment, and quantification of main antioxidant aglycones of flavonoids and tannins from Melastoma dodecandrum lour.: guided by UPLC-ESI-MS/MS," Journal of Chemistry, vol. 2019, pp. 1-12, 2019.

[25] K. J. Marsh, I. R. Wallis, C. Kulheim et al., "New approaches to tannin analysis of leaves can be used to explain in vitro biological activities associated with herbivore defence," New Phytologist, vol. 225, no. 1, pp. 488-498, 2020.

[26] G. Williamson and A. Carughi, "Polyphenol content and health benefits of raisins," Nutrition Research, vol. 30, no. 8, pp. 511-519, 2010.

[27] E. Cantos, J. C. Espín, C. López-Bote, L. De La Hoz, J. A. Ordóñez, and F. A. Tomás-Barberán, "Phenolic compounds and fatty acids from acorns (quercusspp.), the main dietary constituent of free-ranged iberian pigs," Journal of Agricultural and Food Chemistry, vol. 51, no. 21, pp. 62486255, 2003.

[28] A. Glabasnia and T. Hofmann, "Sensory-directed identification of taste-active ellagitannins in American (Quercus albaL.) and European oak wood (Quercus roburL.) and quantitative analysis in bourbon whiskey and oak-matured red wines," Journal of Agricultural and Food Chemistry, vol. 54, no. 9, pp. 3380-3390, 2006.

[29] G. Masson, M. Moutounet, and J. Puech, "Ellagitannin content of oak wood as a function of species and of sampling position in the tree," American Journal of Enology and Viticulture, vol. 46, no. 2, pp. 262-268, 1995.

[30] C. Viriot, A. Scalbert, C. L. M. Hervé du Penhoat, and M. Moutounet, "Ellagitannins in woods of sessile oak and sweet chestnut dimerization and hydrolysis during wood ageing," Phytochemistry, vol. 36, no. 5, pp. 1253-1260, 1994.

[31] E. Cadahía, S. Varea, L. Muñoz, B. Fernández de Simón, and M. C. García-Vallejo, "Evolution of ellagitannins in Spanish, French, and American oak woods during natural seasoning and toasting," Journal of Agricultural and Food Chemistry, vol. 49, no. 8, pp. 3677-3684, 2001. 